\title{
Fast calculation software for modified Look-Locker inversion recovery (MOLLI) T1 mapping
}

Yoon-Chul Kim', Khu Rai Kim², Hyelee Lee ${ }^{3}$ and Yeon Hyeon Choe ${ }^{4^{*}}$ (1)

\begin{abstract}
Background: The purpose of this study was to develop a software tool and evaluate different T1 map calculation methods in terms of computation time in cardiac magnetic resonance imaging.

Methods: The modified Look-Locker inversion recovery (MOLLI) sequence was used to acquire multiple inversion time (TI) images for pre- and post-contrast T1 mapping. The T1 map calculation involved pixel-wise curve fitting based on the T1 relaxation model. A variety of methods were evaluated using data from 30 subjects for computational efficiency: MRmap, python Levenberg-Marquardt (LM), python reduced-dimension (RD) non-linear least square, $C++$ single- and multi-core $\mathrm{LM}$, and $\mathrm{C}++$ single- and multi-core RD.

Results: Median (interquartile range) computation time was $126 \mathrm{~s}$ (98-141) for the publicly available software MRmap, $261 \mathrm{~s}$ (249-282) for python LM, $77 \mathrm{~s}$ (74-80) for python RD, $3.4 \mathrm{~s}(3.1-3.6)$ for C++ multi-core LM, and $1.9 \mathrm{~s}$ (1.9-2.0) for $\mathrm{C}++$ multi-core RD. The fastest $\mathrm{C}++$ multi-core $\mathrm{RD}$ and the publicly available MRmap showed good agreement of myocardial T1 values, resulting in $95 \%$ Bland-Altman limits of agreement of ( -0.83 to $0.58 \mathrm{~ms})$ and ( -6.57 to $7.36 \mathrm{~ms}$ ) with mean differences of $-0.13 \mathrm{~ms}$ and $0.39 \mathrm{~ms}$, for the pre- and post-contrast, respectively.

Conclusion: The $\mathrm{C}++$ multi-core RD was the fastest method on a regular eight-core personal computer for pre- or post-contrast T1 map calculation. The presented software tool (fT1fit) facilitated rapid T1 map and extracellular volume fraction map calculations.
\end{abstract}

Keywords: MRI, Heart, T1 mapping, Parameter estimation

\section{Background}

Cardiac T1 mapping in magnetic resonance imaging (MRI) is a non-invasive and quantitative method for the characterization of the myocardial tissue [1-4] and is particularly useful for the evaluation of diffuse myocardial fibrosis [5]. It typically involves two separate image acquisitions: native T1 mapping (a.k.a. pre-contrast T1 mapping) and post-contrast T1 mapping. Extracellular

\footnotetext{
*Correspondence: ychoe11@gmail.com

${ }^{4}$ Department of Radiology and HVSI Imaging Center, Heart Vascular Stroke Institute, Samsung Medical Center, Sungkyunkwan University School of Medicine, 81 Ilwon-ro, Gangnam-gu, Seoul 06351, South Korea Full list of author information is available at the end of the article
}

volume fraction (ECV), which is a biomarker for myocardial fibrosis, can be attained in a pixel-wise manner from the pre- and post-contrast T1 maps [6, 7]. Due to its quantitative nature, cardiac T1 mapping is advantageous over late gadolinium enhanced imaging, in which the accurate nulling of the healthy myocardial signals in an inversion recovery sequence is challenging in patients with diffuse myocardial fibrosis. The pattern of diffuse myocardial fibrosis is typically observed in patients with non-ischemic heart disease, such as hypertrophic cardiomyopathy $(\mathrm{HCM})$, cardiac amyloidosis, and dilated cardiomyopathy [5]. 
T1 map calculation involves curve fitting for the quantification of $\mathrm{T} 1$ longitudinal relaxation time on a pixelwise basis. The curve fitting process is time-consuming in general, and low-level programming languages such as $\mathrm{C}$ and $\mathrm{C}++$ are desirable for improved computational efficiency. T1 parameters are estimated via non-linear least squares, and Levenberg-Marquardt (LM) optimization is typically utilized with good initial values of the parameters in the T1 fitting model. Alternatively, to overcome the issue of initialization of the parameters and to reduce the search space, Barral et al. presented a reduced dimension non-linear least squares (RD-NLS) approach, which resulted in initialization-free optimization and acceleration in T1 map calculation [8]. In other studies, T1 map calculation was reported to take longer than a minute per image $[9,10]$. Recent related studies of software development in parameter mapping focused on magnetization transfer imaging [11] and neuroimage processing [12], and they lack the comparison of computational efficiency among different calculation methods.

The existence of a variety of methods for T1 map calculation motivated us to develop a software tool for evaluating the performance of the methods. In particular, we sought to develop a Python-based user interface that can also test a $\mathrm{C}++$ implementation with pybind11 [13].
The interface setup facilitates the comparison between Python-based and C++-based methods. Moreover, the Python language serves as a framework for deep learning libraries [14-16] and is commonly adopted for the development of deep learning algorithms, which may have potential for improving the performance in cardiac T1 mapping [17]. In an earlier study, we demonstrated fast T1 map calculation using the LM-based method implemented in $\mathrm{C}++$, as a module for a comprehensive quantitative cardiac MRI analysis tool [18]. In the present study, we focus on evaluating the performance of different T1 map calculation methods with an emphasis on the comparison between the LM method and the RD-NLS method implemented in $\mathrm{C}++$ as well as an emphasis on the comparison between the RD-NLS method and the publicly available MRmap for T1 estimation accuracy in the myocardium.

\section{Methods}

We describe the T1 mapping sequence parameters, T1 map calculation methods, and their implementations and evaluations. Figure 1 shows a custom user interface tool for the study, which is available at https://sites .google.com/site/yoonckim1/software/t1_map_compare.

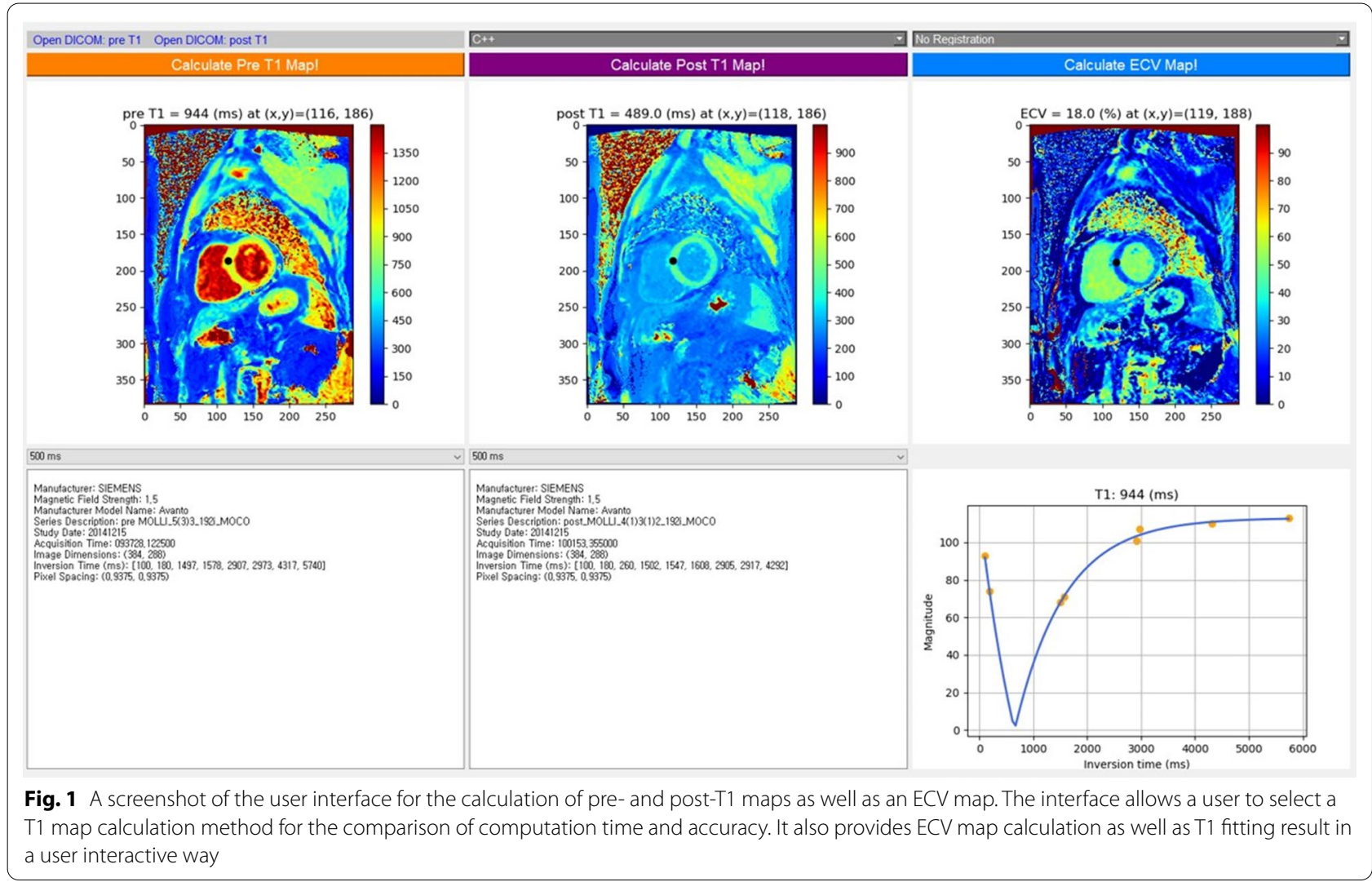


Source code is available at https://github.com/prime52/ fT1fit.

\section{Data acquisition}

Cardiac MRI scans were performed on a $1.5 \mathrm{~T}$ scanner (Siemens Avanto, Erlangen, Germany). Clinical MR examinations were approved by our institutional review board, and informed consent was obtained from the subjects prior to MRI scans. Subjects with suspected cardiovascular diseases were enrolled between May 2014 and May 2015. Subjects who took cardiac pre- and postcontrast T1 mapping scan exams were included for the present study. Subjects with inadequate image quality due to severe motion were excluded. A total of 30 subjects were considered for our study. They consisted of 9 HCM patients, 10 cardiac amyloidosis patients, 7 coronary artery disease patients, and 4 healthy volunteers.

The modified Look-Locker inversion recovery (MOLLI) sequence was used for cardiac T1 mapping $[19,20]$. Imaging parameters were slice thickness $=8 \mathrm{~mm}$, echo time $(\mathrm{TE})=1.01 \mathrm{~ms}$, spacing between slices $=20 \mathrm{~mm}$, the number of phase encoding steps $=104$, pixel bandwidth $=1085 \mathrm{~Hz}$, acquisition matrix $=192 \times 120$, image matrix $=384 \times 288$, pixel spacing $=0.9375 \mathrm{~mm} \times 0.9375 \mathrm{~mm}$, and field of view $(\mathrm{FOV})=360 \mathrm{~mm} \times 270 \mathrm{~mm}$. The MOLLI protocols used were different for the pre-contrast and post-contrast T1 mapping. The MOLLI 5(3)3 protocol used for precontrast $\mathrm{T} 1$ mapping consisted of 5 inversion time (TI) image acquisitions after the first inversion pulse, a threeheartbeat pause for the recovery of the longitudinal magnetization, and 3 TI image acquisitions after the second inversion pulse. The MOLLI 4(1)3(1)2 protocol used for post-contrast T1 mapping consisted of 4 TI image acquisitions after the first inversion pulse, a one-heartbeat pause, 3 TI image acquisitions after the second inversion, a one-heartbeat pause, and 2 TI image acquisitions after the third inversion. Since the post-contrast T1 relaxation time is approximately less than $500 \mathrm{~ms}$, which is much shorter than the pre-contrast T1 ( $950 \mathrm{~ms}$ for myocardium and $1500 \mathrm{~ms}$ for blood), the 3 inversion pulses used in the 4(1)3(1)2 protocol enables more adequate sampling of the early part of $\mathrm{T} 1$ relaxation than the 2 inversion pulses used in the 5(3)3 protocol. Instead of 5 images after the first inversion, 4 images were acquired in the post-contrast $\mathrm{T} 1$ mapping because for the short $\mathrm{T} 1(<500 \mathrm{~ms})$ relaxation the image from the fifth heartbeat appears similar to the image from the fourth heart beat due to fast T1 recovery and thus is unnecessary [21]. The TI images were acquired in a diastolic cardiac phase. All of the TI images were aligned using the motion correction algorithm [22]. The TI images were exported as digital imaging and communications in medicine (DICOM) files for T1 map calculation.

\section{T1 Map calculation}

T1 map calculation was performed pixel-by-pixel. In general, the TI images can be available as either complexvalued or magnitude-valued. In the present study, the DICOM dataset was available as magnitude TI images, and most cardiac MRI scanner systems in hospitals save the DICOM dataset in the magnitude scale by default. At each voxel's location $(\mathrm{x}, \mathrm{y})$, the signal intensity $S(t)$ can be modeled as the following $\mathrm{T} 1$ relaxation curve.

$$
S(t)=a\left(1-e^{-b t}\right)+c
$$

For a set of inversion times $t=\left[T I_{1}, T I_{2}, \ldots, T I_{N}\right]$ and a set of corresponding signals $\left[S\left(T I_{1}\right), S\left(T I_{2}\right), \ldots, S\left(T I_{N}\right)\right]$, there are $N$ equations and 3 unknowns, which are $a, b$, and $c$. In the present study, $N$ was 8 for the pre-contrast data, while $N$ was 9 for the post-contrast data. Notably, $b$ is the reciprocal of $\mathrm{T} 1 *$ (i.e., the apparent $\mathrm{T} 1$ ). The objective function $f$ to be minimized was then given as the sum of squares of the difference between the relaxation model and the data.

$$
f(a, b, c)=\sum_{i=1}^{N}\left(S\left(T I_{i}\right)-a\left(1-e^{-b T I_{i}}\right)-c\right)^{2}
$$

This is a nonlinear least squares problem, and the Levenberg-Marquardt (LM) algorithm [23] can be used to iteratively estimate the parameters $a, b$, and $c$. We empirically selected the initial values for the parameter set $(a$, $b, c)=(350,0.001,-150)$ for the pre-contrast data and $(350,0.005,-150)$ for the post-contrast data. Since our dataset was in the magnitude scale and $S(t)$ of Eq. (1) can cover the negative-value range, we incrementally flipped the polarity of $S\left(T I_{i}\right)$ to find the best fit of Eq. (1) [24]. Notably, the estimated $\mathrm{T}^{*}$ does not incorporate the effect of the tip down of the spin magnetization for every repetition time. Hence, the Look-Locker correction was applied in the following way to arrive at the corrected $\mathrm{T} 1$ value:

$$
T 1=T 1^{*}\left(\frac{a}{a+c}-1\right)
$$

Meanwhile, the RD-NLS (or RD) method expands the objective function Eq. (2) and finds the optimal estimates of the parameters separately (refer to Appendix B in Barral et al. [8] for detailed mathematical derivations). The function to be optimized for $\mathrm{T} 1$ is independent of the other two parameters. Hence, the decoupling leads to a one-dimensional search problem for $\mathrm{T} 1$ estimation. 


\section{Python and $\mathrm{C}++$ implementation}

For the LM method, we implemented the T1 map calculation in Python. To solve the non-linear least squares problem, we used the scipy.optimize.curve_fit() function, which is based on the Levenberg-Marquardt algorithm. The method is referred to as LM_python. For the RD method, we converted the original MATLAB script written by Barral et al. (code available at http://www-mrsrl.stanford.edu/ jbarral/t1map.html) to Python. We noted that the original RD algorithm failed to work in some cases where the two candidate TIs produced unsatisfactory fitting results. Hence, we modified the RD algorithm by introducing three candidate TIs. This helped remove the unsatisfactory fitting and improved the accuracy (Fig. 2). The method is referred to as RD_python.

We also implemented both the LM- and RD-based T1 map calculations in $\mathrm{C}++$ and used pybind11 [13] to make the compiled $\mathrm{C}++$ code compatible with the Python environment. The $\mathrm{C}++$ implementation was performed on a Windows OS, Microsoft Visual Studio 2017 platform. For the LM-based T1 parameter estimation, we used the solve_least_squares_lm function of the Dlib library [25]. In addition, for the multi-core implementation, we used the OpenMP library [26] for the parallelization of the 'for' loop in the pixel-wise T1 map calculation. We chose the static schedule option for parallelization. For evaluation, the methods are referred to
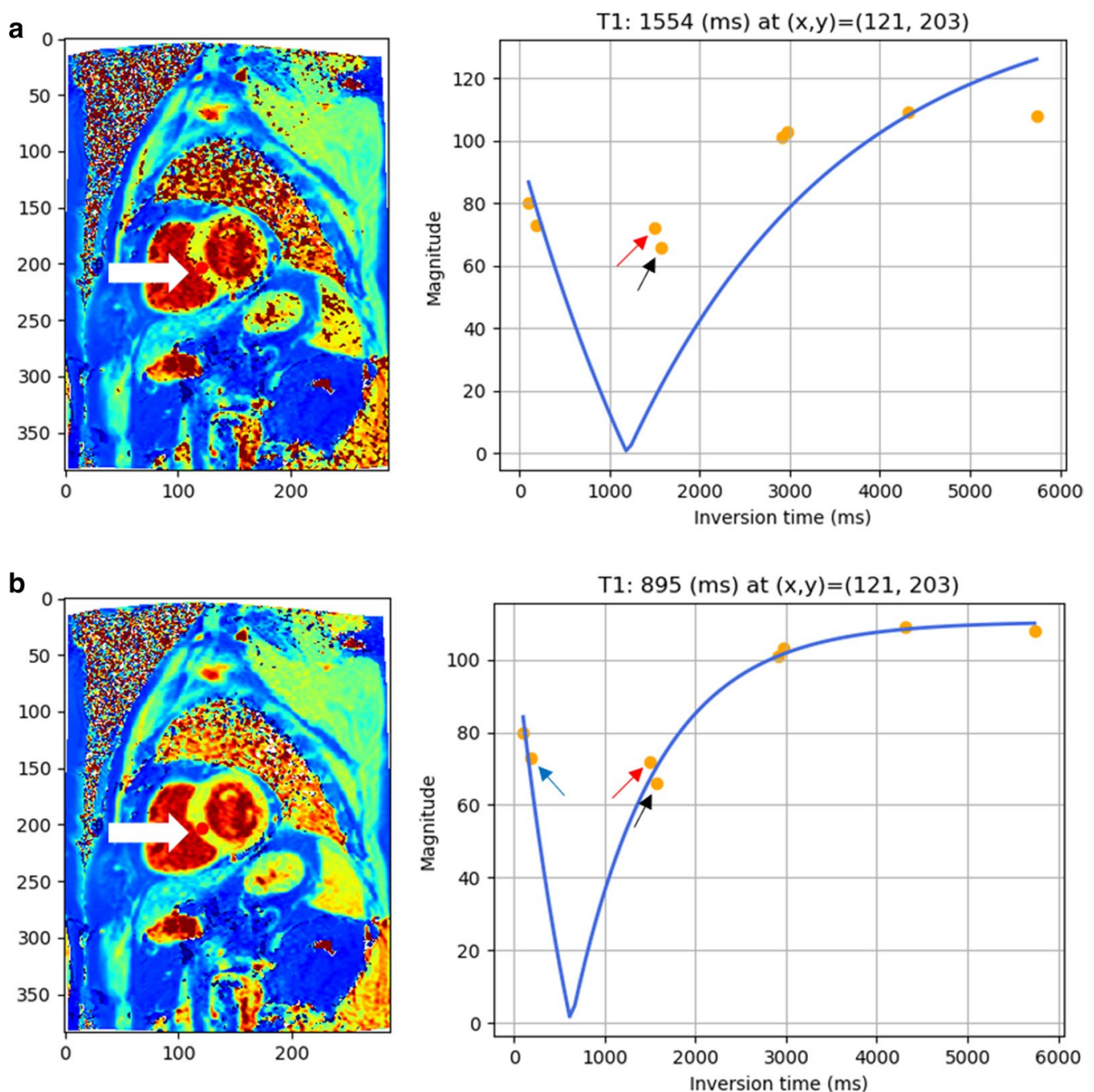

Fig. 2 An example of improved fitting result after modification of the original RD-NLS method. The unsatisfactory performance of the original RD-NLS occurred when some inversion time ( $\mathrm{TI}$ ) values were very close such that the choice of minimal TI closest to 0 can be sensitive to noise. a A poor fitting result when the RD-NLS method was applied without any modification of the script. $\mathbf{b}$ A correction after the modification, which considered three candidate samples for polarity restorations (see arrows) 
as LM_C ++ _single-core, RD_C ++ +single-core, LM $\mathrm{C}++$ multi-core, and $\mathrm{RD} C++$ multi-core.

To ensure that the curve fitting is correctly performed at each pixel location and the samples are placed close to the $\mathrm{T} 1$ relaxation curve, we developed a graphical user interface (GUI) that enables a user to load the DICOM TI image data, select a method for T1 map calculation, and mouse-click a pixel location for displaying its curve fitting result. The GUI also performs the calculation and display of the pre- and post-contrast T1 maps as well as the ECV map (Fig. 1). The GUI was implemented using the PyQT library [27] on a 64-bit Windows PC.

\section{Evaluation}

We evaluated the following methods in terms of speed on a Windows PC (AMD Ryzen 7 1800X Eight-Core Processor and 16.0 GB RAM): MRmap, LM_python, RD_ python, LM_C++_single-core, RD_C++_single-core, LM_C++_multi-core, and RD_C++_multi-core.

For the evaluation, we used the MRmap software [9], which is publicly available for download at https:// sourceforge.net/projects/mrmap/. We chose the following options for T1 map calculation: Limits of T1, T2, and Noise for pre-contrast were set to 3000, 350, and 0, respectively. Limits of T1, T2, and Noise for post-contrast were set to 1500,350 , and 0 , respectively. Specifically, the setting of the Noise value had to be consistent, since the choice of the value significantly affected the computation speed. Registration was set to None. Process was set to "T1 mapping-MOLLI", and Correction was set to "Look-Locker."

For the evaluation of accuracy in T1 value, we manually drew a region of interest (ROI) in the myocardium in either a pre- or a post-contrast T1 map of each subject and used the same ROI mask for the T1 maps estimated using MRmap and the RD_C++_multi-core method. Mean T1 values were calculated within the myocardial ROIs. Bland-Altman analysis was performed by computing the mean difference and $95 \%$ limits of agreement between the two T1 measurements.

\section{Results}

Table 1 lists the computation time for MRmap, LM_python, RD_python, LM_C++_single-core, $\mathrm{RD}$-C++_single-core, LM_C++_multi-core, and RD_ $\mathrm{C}++$ multi-core for pre- and post-contrast TI image sets in 30 subjects. The RD method was superior to the LM method in computation time for all three different ways of implementation: python, single-core $\mathrm{C}++$, and multi-core $\mathrm{C}++$. The RD_C++_multi-core method took approximately $2 \mathrm{~s}$ for $\mathrm{T} 1$ map generation in both pre- and post-contrast T1 maps. There were statistically

\begin{tabular}{|c|c|c|}
\hline Method & Pre-contrast & Post-contrast \\
\hline MRmap & $126.0(98.3-140.6)$ & $111.8(93.7-140.6)$ \\
\hline LM_python & $261.3(249.3-282.4)$ & $249.6(242.6-262.1)$ \\
\hline RD_python & $77.0(74.0-80.1)$ & $77.8(75.9-81.4)$ \\
\hline LM_C++_single-core & $28.6(27.3-29.8)$ & $28.0(26.9-29.9)$ \\
\hline RD_C++_single-core & $15.2(15.0-15.9)$ & $16.2(16.1-16.8)$ \\
\hline LM_C++_multi-core* & $3.4(3.1-3.6)$ & $3.3(3.0-3.5)$ \\
\hline RD_C++_multi-core* & $1.9(1.9-2.0)$ & $2.1(2.0-2.2)$ \\
\hline
\end{tabular}

* Eight cores were simultaneously used for the calculation

significant differences in computation time between RD_C++_multi-core and LM_C++_multi-core: $1.9 \mathrm{~s}$ vs. $3.4 \mathrm{~s}(\mathrm{p}<0.001)$ for pre-contrast and $2.1 \mathrm{~s}$ vs. $3.3 \mathrm{~s}$ $(\mathrm{p}<0.001)$ for post-contrast. The distributions of computation time clearly exhibit the superior performance of RD_C++_multi-core (Fig. 3).

Qualitative comparisons of the methods in preand post-contrast T1 measurements are shown in an HCM patient (Fig. 4), and all the methods produced similar T1 measurements in the myocardium and blood regions of interest. These similarities were also observed in other subjects' T1 map data. Curve fitting examples for the pre-contrast and post-contrast MOLLI protocols are shown for the blood and myocardium in a normal volunteer (Fig. 5). In particular, the post-contrast MOLLI 4(1)3(1)2 protocol, which uses three inversion pulses, is advantageous in estimating a short $\mathrm{T} 1$ value by fitting three samples in early TIs, over the pre-contrast MOLLI 5(3)3 protocol, which uses two inversion pulses.

T1 measurements in the myocardium for the four methods (i.e., LM_python, RD_python, LM_C++, RD_ $\mathrm{C}++$ ) are shown in Fig. 6 for the pre-contrast (top row) and post-contrast (bottom row) cases. The Bland-Altman plots show good agreement between each of the four methods and the reference MRmap in all subjects except for a few outliers. Most of the T1 difference values lie within $2 \mathrm{~ms}$. Table 2 shows Bland-Altman statistics for the four methods. For all the methods, the absolute mean difference was small $(<1 \mathrm{~ms})$ for the preand post-contrast cases. The $95 \%$ limit of agreement was wider for the post-contrast than for the pre-contrast, but this is likely due to the outlier whose difference value was approximately $18 \mathrm{~ms}$ (see the plots in the bottom row of Fig. 6). As expected, the amyloidosis group (AMYL) shows high pre-contrast $\mathrm{T} 1$ values in the myocardium (see the plots in the top row of Fig. 6). 

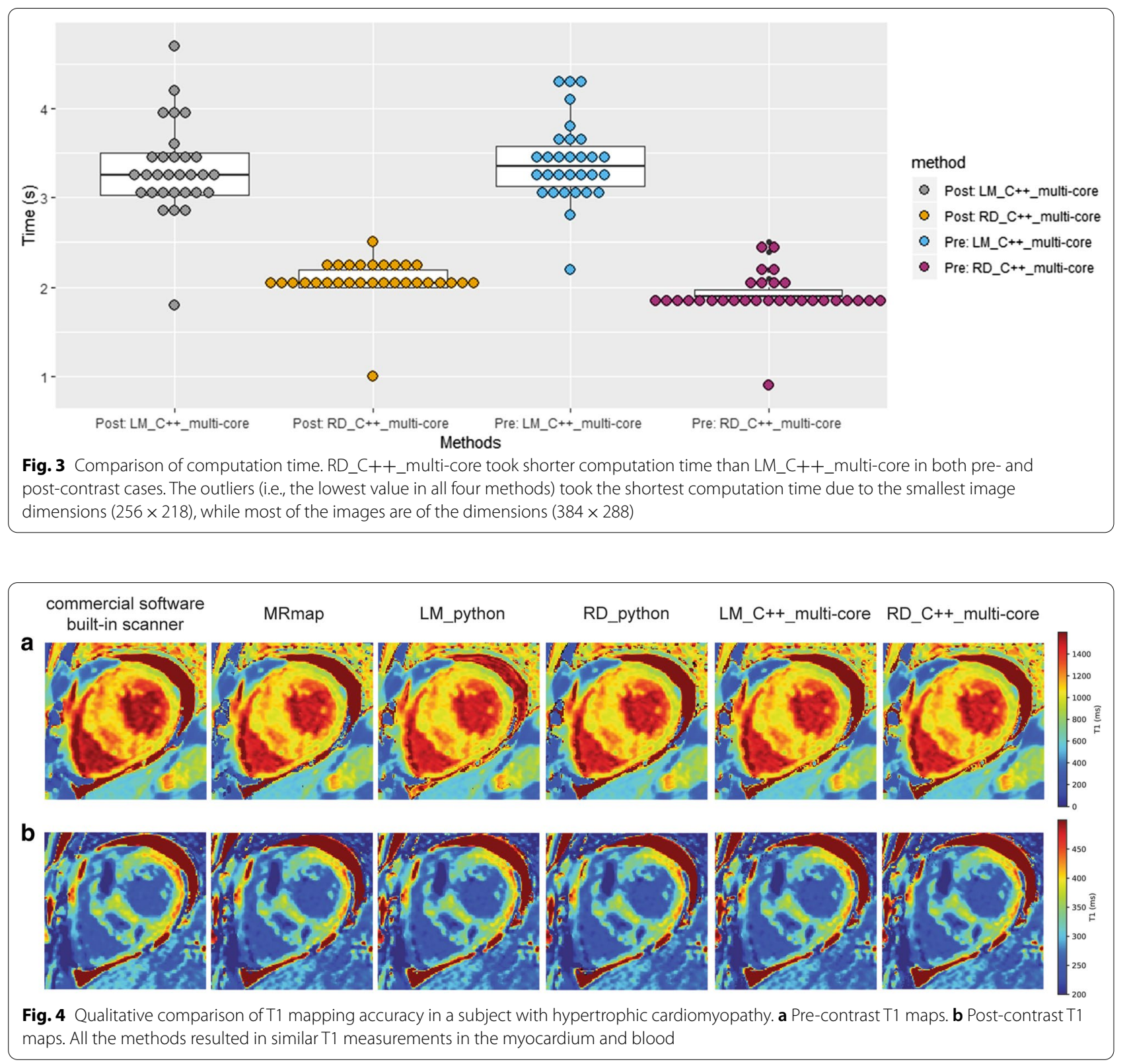

\section{Discussion}

We demonstrated a rapid T1 map calculation method, which only took approximately $2 \mathrm{~s}$ for $384 \times 288 \times 8$ or $384 \times 288 \times 9$ images on a personal desktop computer equipped with an eight-core processor. This is a significant improvement over other methods demonstrated in the literature. For comparison, we measured computation time on MRmap, which was $126 \mathrm{~s}$ in pre-contrast and $112 \mathrm{~s}$ in post-contrast. In the literature, MRmap was reported to take $113 \mathrm{~s}$ for a set of $128 \times 128 \times 8$ images with a noise level of 0 [9]. The MRmap code was written in Interactive Data Language (IDL), which is a high-level language so that it is computationally slow. Other software tools also deserve to be mentioned. The T1 map calculation of Altabella et al. was reported to take $66 \mathrm{~s}$ for the magnitude fitting on 31,080 fitted voxels from a set of $218 \times 256 \times 8$ images [10]. The T1 map calculation of Liu et al., referred to as the vectorized Levenberg-Marquardt fitting, was reported to take $60 \mathrm{~s}$ on average in MATLAB for a set of $256 \times 256 \times 8$ images. It is undeniable that the $\mathrm{C}++$ nature of the proposed method was the main cause of the speed improvement. However, it is important to note that the way of implementation via the Python wrapper 


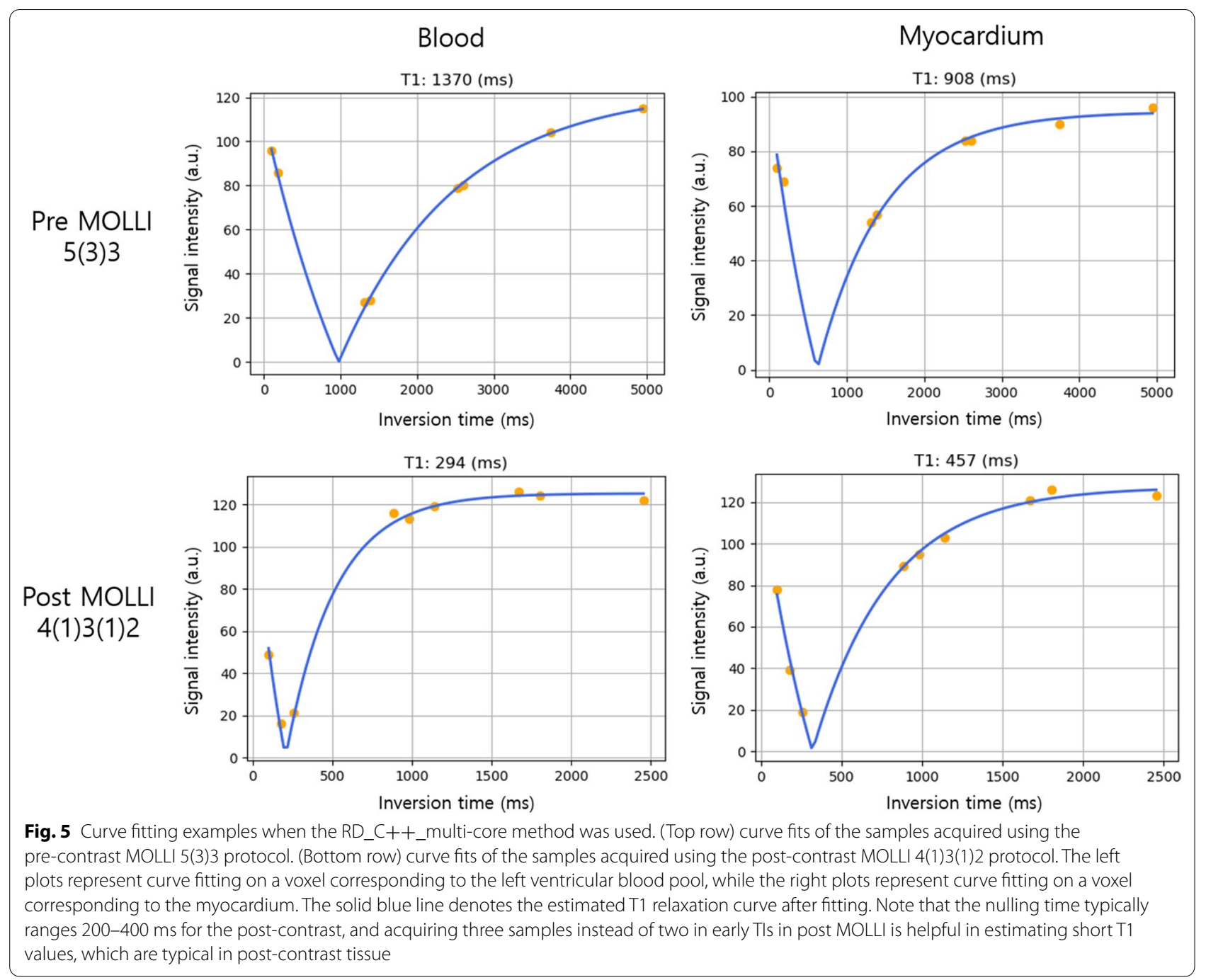

facilitated code readability and maintenance. It is noted that a $\mathrm{C}++$ implementation for $\mathrm{T} 1$ mapping was recently demonstrated by Werys et al. [28], although we did not compare their method with our methods.

There may be room for improvement in computational speed in the proposed method. First, the noise level (or the threshold level) can be applied to the images as was demonstrated in $[9,10]$. By increasing the threshold up to an acceptable level, one can exclude a fairly large number of pixels whose intensity is not sufficiently high (e.g., the background air region) from the curve fitting process, and this could help reduce the computation time. Second, loop scheduling can be optimized when using the OpenMP library. In general, OpenMP provides three kinds of scheduling: static, dynamic, and guided. There may be an opportunity for speed improvement by, for example, trying these scheduling methods with different choices of chunk size in dynamic mode and finding the optimal one.

Python provides a convenient environment for developers in terms of code readability and thus facilitates the debugging process. This was especially true in the design of a T1 map calculation algorithm and a graphical user interface in the present study. We first implemented a Python version of the T1 map calculation and translated it to a $\mathrm{C}++$ version. For the verification of the $\mathrm{C}++$ implementation in terms of accuracy, we compared the T1 maps generated by the Python implementation and the $\mathrm{C}++$ method within the graphical user interface. This was helpful in evaluating the accuracy of the $\mathrm{C}++$ implementation.

In this study, different MOLLI sequence protocols were used in the acquisitions of pre-contrast and postcontrast T1 data: MOLLI 5(3)3 for the pre-contrast and MOLLI 4(1)3(1)2 for the post-contrast. There are other 

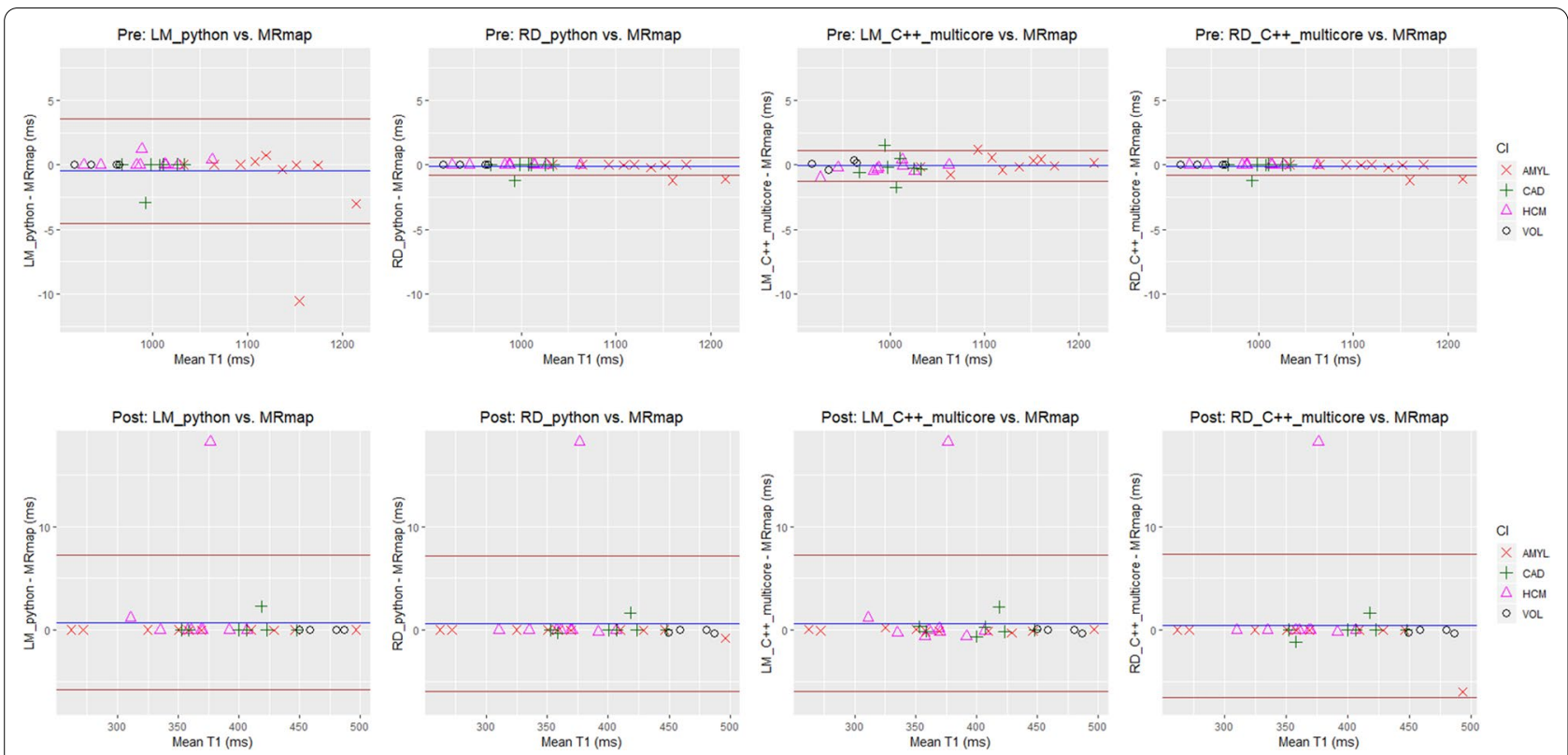

Fig. 6 Bland-Altman plots for the four $T 1$ calculation methods when compared with MRmap as reference. (Top row) pre-contrast $T 1$ measurements in the myocardium. (Bottom row) post-contrast T1 measurements in the myocardium. From left to right, (1) LM_python, (2) RD_python, (3) LM_ C++_multi-core, and (4) RD_C++_multi-core

Table 2 Bland-Altman statistics for the myocardial T1 measurements with MRmap as the reference method

\begin{tabular}{|c|c|c|c|c|}
\hline & \multicolumn{2}{|l|}{ Pre-contrast } & \multicolumn{2}{|l|}{ Post-contrast } \\
\hline & Mean Difference, $\mathrm{ms}$ & $95 \%$ Limits of Agreement, $\mathrm{ms}$ & Mean Difference, ms & $\begin{array}{l}\text { 95\% Limits } \\
\text { of Agreement, } \\
\text { ms }\end{array}$ \\
\hline LM_python & -0.24 & -5.11 to 4.63 & 0.72 & -5.81 to 7.25 \\
\hline RD_python & -0.13 & -0.83 to 0.58 & 0.60 & -5.95 to 7.14 \\
\hline LM_C++_multi-core ${ }^{*}$ & -0.04 & -1.34 to 1.25 & 0.63 & -5.96 to 7.22 \\
\hline RD_C++_multi-core* & -0.13 & -0.83 to 0.58 & 0.39 & -6.57 to 7.36 \\
\hline
\end{tabular}

* T1 measurements between multi-core and single-core were the same

MOLLI sequence variants reported in the literature (Table 2 of Kellman and Hansen [20]). Intra-individual comparisons among the MOLLI sampling schemes may be worth investigating because the curve fitting on different sampling schemes may affect the T1 map results as well as the ECV map results. However, implementing the intra-individual comparisons would be challenging due to patient discomfort as a result of repeated use of contrast agent and repeated breath-holds.

The rapid computation framework based on pybind 11 and multicore $\mathrm{C}++$ has the potential to be applied to three-dimensional (3D) T1 mapping [29], which has larger dimensions than 2D T1 mapping, as well as to other pixel-wise parameter estimation of $\mathrm{T} 2$, $\mathrm{T} 2 *$, tissue perfusion, and permeability. For example, deconvolution process in model-based tissue perfusion quantification [30] can be accelerated using $\mathrm{C}++$ and parallel processors.

The current study has several limitations. First, this was a single-center study conducted using data acquired on the Siemens $1.5 \mathrm{~T}$ scanner, with a small number of subjects $(n=30)$ for evaluation. Second, the tool supported only the magnitude data rather than both magnitude and complex-valued data. Third, the computation time was assessed on a single desktop computer only. Evaluation on other types of computers (e.g., mobile phone with a restricted capacity for central processing unit (CPU) and memory, workstation computer with a higher capacity for CPU and memory) would be worth investigating. 


\section{Conclusions}

We evaluated the performance of the RD-NLS in terms of speed and accuracy compared with that of the LM method. The RD-NLS implemented with $\mathrm{C}++$ and parallel processing library was the fastest, taking $<3 \mathrm{~s}$ in both pre- and post-contrast T1 map calculation on a regular desktop computer. The Python wrapper has the potential to improve workflow in the implementation of rapid pixelwise parametric mapping, not merely of T1 estimation but also of tissue perfusion- and permeability-related parameter estimation. The implementation details available as open source may be helpful resources for other researchers' investigations and validation of new methods in parameter mapping.

\begin{abstract}
Abbreviations
MRI: Magnetic resonance imaging; ECV: Extracellular volume fraction; LM: Levenberg-Marquardt; RD-NLS: Reduced dimension non-linear least squares; MOLLI: Modified Look-Locker inversion recovery; TE: Echo time; FOV: Field of view; TI: Inversion time; DICOM: Digital imaging and communications in medicine; GUI: Graphical user interface; ROI: Region of interest; HCM: Hypertrophic cardiomyopathy; AMYL: Amyloidosis; CAD: Coronary artery disease; VOL: Healthy volunteer; CPU: Central processing unit.
\end{abstract}

\section{Acknowledgements}

Not applicable.

\section{Author contributions}

YK, KRK, HL implemented the methods. YK analyzed the results of the methods. YK was a main contributor in writing the manuscript. YHC designed the study and drafted the manuscript. All authors have read and approved the manuscript.

\section{Funding}

This work was supported by the National Research Foundation of Korea (grant number 2018R1D1A1B07042692) and the DongKook Life Science. Co., Ltd. Republic of Korea. The funding sources had no role in the study design, data collection, and data analysis or in the writing of the manuscript.

\section{Availability of data and materials}

Data in the present study are not publicly available since they include private patient information. The de-identified data are available from the corresponding author upon request after the approval of the institutional review board of Samsung Medical Center. The code is available at https://github.com/prime 52/fT1fit.

\section{Ethics approval and consent to participate}

This study was approved by the institutional review board at Samsung Medical Center, and the requirement for informed consent for the use of patient data was waived.

\section{Consent for publication}

Not applicable.

\section{Competing interests}

The authors declare that they have no competing interests.

\section{Author details}

${ }^{1}$ Clinical Research Institute, Samsung Medical Center, Sungkyunkwan University School of Medicine, Seoul, South Korea. ${ }^{2}$ Department of Electronic Engineering, Sogang University, Seoul, South Korea. ${ }^{3}$ Department of Mathematics, Sogang University, Seoul, South Korea. ${ }^{4}$ Department of Radiology and HVSI Imaging Center, Heart Vascular Stroke Institute, Samsung Medical Center, Sungkyunkwan University School of Medicine, 81 Ilwon-ro, Gangnam-gu, Seoul 06351, South Korea.
Received: 14 July 2020 Accepted: 2 February 2021

Published online: 12 February 2021

\section{References}

1. Haaf P, Garg P, Messroghli DR, Broadbent DA, Greenwood JP, Plein S. Cardiac T1 Mapping and Extracellular Volume (ECV) in clinical practice: a comprehensive review. J Cardiovasc Magn Resonance. 2016;18(1):89. https://doi.org/10.1186/s12968-016-0308-4.

2. Moon JC, et al. Myocardial T1 mapping and extracellular volume quantification: a Society for Cardiovascular Magnetic Resonance (SCMR) and CMR Working Group of the European Society of Cardiology consensus statement. J Cardiovasc Magn Resonance. 2013;15:92. https://doi. org/10.1186/1532-429X-15-92.

3. Puntmann VO, Peker E, Chandrashekhar Y, Nagel E. T1 mapping in characterizing myocardial disease: a comprehensive review. Circ Res. 2016;119(2):277-99. https://doi.org/10.1161/CIRCRESAHA.116.307974.

4. Jo Y, et al. Guidelines for cardiovascular magnetic resonance imaging from Korean Society of Cardiovascular Imaging (KOSCl)-Part 1: standardized protocol. Investig MRI. 2019;23(4):296-315.

5. Reiter G, Reiter C, Krauter C, Fuchsjager M, Reiter U. Cardiac magnetic resonance T1 mapping. Part 1: aspects of acquisition and evaluation. Eur J Radiol. 2018;109:223-34. https://doi.org/10.1016/j.ejrad.2018.10.011.

6. Schelbert EB, Messroghli DR. State of the art: clinical applications of cardiac T1 mapping. Radiology. 2016;278(3):658-76. https://doi.org/10.1148/ radiol.2016141802.

7. Cameron D, Vassiliou VS, Higgins DM, Gatehouse PD. Towards accurate and precise 1 and extracellular volume mapping in the myocardium: a guide to current pitfalls and their solutions. MAGMA. 2018;31(1):143-63. https://doi.org/10.1007/s10334-017-0631-2.

8. Barral JK, Gudmundson E, Stikov N, Etezadi-Amoli M, Stoica P, Nishimura DG. A robust methodology for in vivo T1 mapping. Magn Reson Med. 2010;64(4):1057-67. https://doi.org/10.1002/mrm.22497.

9. Messroghli DR, et al. An open-source software tool for the generation of relaxation time maps in magnetic resonance imaging. BMC Med Imaging. 2010;10:16. https://doi.org/10.1186/1471-2342-10-16.

10. Altabella $L$, et al. A feasible and automatic free tool for $T 1$ and $E C V$ mapping. Phys Med. 2017;33:47-55. https://doi.org/10.1016/j. ejmp.2016.12.002.

11. Cabana JF, et al. Quantitative magnetization transfer imaging made easy with q MTL ab: software for data simulation, analysis, and visualization. Concepts Magn Resonance Part A. 2015;44(5):263-77.

12. Wood TC. QUIT: QUantitative imaging tools. J Open Sour Softw. 2018;3(26):656.

13. Jakob W, Rhinelander J, Moldovan D. pybind11 — Seamless operability between C++11 and Python. 2017. https://github.com/pybind11/pybin d11.

14. Paszke Aet al. Pytorch: An imperative style, high-performance deep learning library. In: Advances in neural information processing systems, 2019; pp. 8026-8037.

15. Abadi $M$, et al., Tensorflow: a system for large-scale machine learning. In: 12th \{USENIX\} symposium on operating systems design and implementation (\{OSDI\} 16), 2016, pp. 265-283.

16. Chollet F. Keras: The python deep learning library. ascl, p. ascl: 1806.022, 2018.

17. Feng L, Ma D, Liu F. Rapid MR relaxometry using deep learning: An overview of current techniques and emerging trends. NMR Biomed. 2020 https://doi.org/10.1002/nbm.4416.

18. Kim YC, Kim KR, Choi K, Kim M, Chung Y, Choe YH. EVCMR: a tool for the quantitative evaluation and visualization of cardiac MRI data. Comput Biol Med. 2019;111:103334. https://doi.org/10.1016/j.compbiomed .2019.103334.

19. Messroghli DR, Radjenovic A, Kozerke S, Higgins DM, Sivananthan MU, Ridgway JP. Modified Look-Locker inversion recovery (MOLLI) for highresolution T1 mapping of the heart. Magn Reson Med. 2004;52(1):141-6. https://doi.org/10.1002/mrm.20110.

20. Kellman P, Hansen MS. T1-mapping in the heart: accuracy and precision. J Cardiovasc Magn Resonance. 2014;16:2. https://doi. org/10.1186/1532-429X-16-2. 
21. Schelbert EB, et al. Myocardial extravascular extracellular volume fraction measurement by gadolinium cardiovascular magnetic resonance in humans: slow infusion versus bolus. J Cardiovasc Magn Resonance. 2011;13:16. https://doi.org/10.1186/1532-429X-13-16.

22. Xue $\mathrm{H}$, et al. Motion correction for myocardial T1 mapping using image registration with synthetic image estimation. Magn Reson Med. 2012;67(6):1644-55. https://doi.org/10.1002/mrm.23153.

23. Nocedal J, Wright S. Numerical optimization. Berlin: Springer; 2006.

24. Bakker CG, De Graaf CN, Van Dijk P. Restoration of signal polarity in a set of inversion recovery NMR images. IEEE Trans Med Imaging. 1984;3(4):197202. https://doi.org/10.1109/TMI.1984.4307681.

25. King DE. Dlib-ml: a machine learning toolkit. J Mach Learn Res. 2009;10:1755-8.

26. Chandra R, Dagum L, Kohr D, Menon R, Maydan D, McDonald J. Parallel programming in OpenMP. London: Morgan Kaufmann; 2001.

27. Summerfield M. Rapid GUI programming with Python and Qt: the definitive guide to PyQt programming. London: Pearson Education; 2007.
28. Werys K, et al. Total Mapping Toolbox (TOMATO): an open source library for cardiac magnetic resonance parametric mapping. SoftwareX, vol. 11 2019

29. Clique H, Cheng HL, Marie PY, Felblinger J, Beaumont M. 3D myocardial T1 mapping at 3T using variable flip angle method: pilot study. Magn Reson Med. 2014;71(2):823-9. https://doi.org/10.1002/mrm.24688.

30. Jerosch-Herold M. Quantification of myocardial perfusion by cardiovascular magnetic resonance. J Cardiovasc Magn Res. 2010;12:57. https://doi. org/10.1186/1532-429X-12-57.

\section{Publisher's Note}

Springer Nature remains neutral with regard to jurisdictional claims in published maps and institutional affiliations.
Ready to submit your research? Choose BMC and benefit from:

- fast, convenient online submission

- thorough peer review by experienced researchers in your field

- rapid publication on acceptance

- support for research data, including large and complex data types

- gold Open Access which fosters wider collaboration and increased citations

- maximum visibility for your research: over $100 \mathrm{M}$ website views per year

At BMC, research is always in progress.

Learn more biomedcentral.com/submissions 